J. Cuesta-Herranz 1 , J.J. Laguna², R. Mielgo 3 , I. Pérez-Camo ${ }^{4}$, A.M. Callejo5, L. Begona ${ }^{6}$, M.C. Gomez ${ }^{6}$, B. Madariaga ${ }^{6}$, A. Martinez ${ }^{6}$

\title{
Quality of life improvement with allergen immunotherapy treatment in patients with rhinoconjunctivitis in real life conditions. Results of an observational prospective study (ÍCARA)
}

\author{
${ }^{1}$ IIS-Fundación Jiménez Díaz, Allergy Department, Madrid, Spain \\ ${ }^{2}$ Allergy Unit, Allergo-Anaesthesia Unit, Hospital Central de la Cruz Roja, Faculty of Medicine, Alfonso X El Sabio Univer- \\ sity, ARADyAL, Madrid, Spain \\ ${ }^{3}$ Hospital 12 de Octubre, Allergy Department, Madrid, Spain \\ ${ }^{4}$ Hospital Royo Villanova, Allergy Department, Zaragoza, Spain \\ ${ }^{5}$ Hospital Virgen de la Concha, Allergy Department, Zamora, Spain \\ ${ }^{6}$ ROXALL Medicina España S.A., R and D Department, Zamudio, Bizkaia, Spain
}

\section{KEY WORDS}

allergic rhinoconjunctivitis; allergen

immunotherapy; AIT; quality of life;

$H R Q o L ; R Q L Q$

\section{Corresponding author}

Leire Begoña

ROXALL Medicina España, S.A.

Parque Científico y Tecnológico de Bizkaia

Edificio 401, 48170 Zamudio, Bizkaia, Spain

Phone: +34944438000

Fax: +34944438016

E-mail: leire.begona@roxall.es

Doi

10.23822/EurAnnACI.1764-1489.104

\begin{abstract}
Summary
Objectives. Evaluate the changes in quality of life of patients with allergic rhinoconjunctivitis (AR), with or without asthma, after one-year treatment with allergen immunotherapy. Methods. This was an observational prospective multicenter study. RQLQ questionnaire and VAS scale to assess treatment satisfaction were used. Impact on $A R$ and asthma was also analyzed. Any adverse reaction was recorded. Results. 127 patients were recruited. Mean values in RQLQ decreased from 2.61 to 1.34 points, reflecting a statistically and clinically significant improvement $(p<$ 0.01). The percentage of asthmatic patients decreased significantly $(p<0.01)$. Mean value of patients' satisfaction was $7.24(S D=1.90)$. Only 11 patients presented systemic reactions (9.17\%), none of them serious. Conclusions. One-year AIT treatment significantly increases QoL in patients with AR. Moreover, high patients' satisfaction values were reported, together with an adequate safety profile.
\end{abstract}

\section{List of abbreviations}

AEMPS, Agencia Española de medicamentos y productos sanitarios; AIT, allergen immunotherapy; AR, allergic rhinoconjunctivitis; ARIA, allergic rhinitis and its impact on asthma; CEIm, Comité de ética de la investigación con medicamentos; GINA, Global initiative for asthma; HRQoL, health-related quality of life; IRB, institutional review board; MID, meaningful importance difference; QoL, quality of life; RQLQ, Rhino- conjunctivitis quality of life questionnaire; SCIT, subcutaneous immunotherapy; SLIT, sublingual immunotherapy; STROBE, Strengthening the reporting of observational studies in epidemiology; VAS, Visual analogue scale.

\section{Introduction}

Allergic rhinoconjunctivitis (AR) is an allergic disorder of the nose and eyes, resulting in a chronic, mostly eosinophilic, in- 
flammation of the nasal mucosa and conjunctiva (1). It is characterized by symptoms of nasal obstruction, watery nasal discharge, sneezing and itching and, when it affects conjunctiva, ocular itching, injection and tearing (2). It is mediated by $\operatorname{IgE}$ antibodies and it is secondary to exposure to offending allergens in previously sensitized patients. Depending on exposure patterns and the nature of the allergen triggers, the symptoms may be intermittent, persistent or persistent with intermittent exacerbations (3). It is considered the most prevalent allergic disease, affecting around $25 \%$ of population in Western Europe (4), and it is frequently associated with other allergic manifestations, both respiratory and otherwise (5). Although AR does not endanger patients' lives, it can result in considerable morbidity $(6,7)$, and can cause a significant deterioration in patients' quality of life (QoL) $(8,9)$. AR is also a risk factor for the development of asthma (10).

The effects of AR on Health-related Quality of Life (HRQoL) extend to learning, sleep, vitality / alertness, perception of general health, cognitive and emotional functioning, and psychomotor performance $(11,12)$. All these possible limitations in patients' day to day can have considerable negative effects on the person's performance both at work or school, and at home, having a direct and indirect economic impact on society $(11,13)$. Symptoms can, in many cases, be controlled with avoidance measures and pharmacological therapies such as oral, intranasal and topical $\mathrm{H} 1$ antihistamines, intranasal corticosteroids and antileukotrienes, as monotherapy, or in combination $(14,9)$. Allergen immunotherapy (AIT) with the subcutaneous or sublingual administration of the causative allergen(s), is an additional potential treatment option, particularly for those patients with more troublesome diseases which remain inadequately controlled despite avoidance measures and regular pharmacotherapy $(15,9)$. The problem of inadequately controlled AR, despite optimal medical treatment, continues to represent a therapeutic challenge in the majority of patients, since consequently a significant number of patients continue to experience symptoms that affect their HRQoL. AIT has also been shown to have a disease-modifying effect (16), since it can not only desensitize a patient, thereby ameliorating symptoms, but also deliver long-term clinical benefits that may persist for years after discontinuation of treatment $(15,17)$. For the above mentioned, nowadays AIT is considered the only etiological treatment of allergic diseases caused by inhalant allergens and Hymenoptera venom $(15,18)$.

Improvements of the disease-specific HRQoL are especially important for long-term treatments like AIT, and assessment of treatment effectiveness in real life is essential. Currently, a number of studies with AIT have been reported, where the improvement in HRQoL is evaluated mostly as a secondary efficacy variable $(19,20,21,22)$. These studies had shown positive results in HRQoL, but most are clinical trials with sublingual immunotherapy (SLIT), and data from real life studies are still scarce $(23,24)$. Therefore, there is still a need for more clinical evidence, specially with non-interventional studies, where a higher representation of patient's population can be included, as children, patients with comorbidities, etc. are not usually included in clinical trials. Moreover, it is also very important to identify possible factors that may be associated with HRQoL improvement, that could help in the decisions of physicians' day-to-day clinical practice.

The main objective of this study was to evaluate the changes in HRQoL in patients with AR with or without asthma, after one-year treatment with ROXALL subcutaneous immunotherapy (SCIT). As secondary objectives, the impact on AR and asthma symptoms, satisfaction reported either by patients and physicians, patients' adherence to treatment and treatment safety were evaluated. Moreover, the identification of possible patient and treatment factors associated with the AIT efficacy were also analyzed.

\section{Material and methods}

This article was written following the Strengthening the Reporting of Observational studies in Epidemiology (STROBE) guidelines (25). The study was a non-interventional prospective multicenter clinical study performed in 13 allergy departments in Spain between June 2015 and May 2017. Patients were included in the study in a baseline visit in which patients' eligibility was checked and the informed consent was signed. Then, the patient started the treatment with the administration of the first dose of SCIT. All patients started the treatments between September 2015 and March 2016, and finalized the study follow-up 1-year after. The baseline RQLQ questionnaire was completed by the patients just before starting treatment, and the final RQLQ questionnaire was carried out after 1 year, corresponding therefore to the same moment during the year as the baseline RQLQ questionnaire was performed. Included patients were those diagnosed of allergic rhinoconjunctivitis, with or without asthma, in which subcutaneous AIT (ROXALL Medicina España S.A.) was prescribed in a routine clinical practice basis, in either formulation, composition or administration schedule. The study was approved by AEMPS (Agencia Española de medicamentos y productos sanitarios) and all the involved regional competent authorities, and by an Institutional review board (IRB) (CEIM hospitales Torrevieja, Elche-Vinalopó) according to Spanish regulation, and other local IRBs. Before participation, all patients gave their signed informed consent.

\section{Patients selection criteria}

The assignment of a patient to a specific AIT treatment was not decided in advance by the study protocol, whereas was decided 
by the physician according to their usual clinical practice, and following EAACI recommendations for the use of AIT with aeroallergens. No intervention either diagnostic or of follow-up was applied to patients, other than the usual clinical practice. Eligible patients were those over 12 years of age suffering from AR with or without asthma, with type I hypersensitivity to one or more aeroallergens, responsible for their clinical manifestations, according to:

- positive result Prick test, defined as: a positive result of at least $3 \mathrm{~mm}$ in diameter for one or more aeroallergens;

- specific IgE value $\geq$ class 2 ( $\geq 0.70 \mathrm{kU} / \mathrm{L}$ ) (CAP/PHADIA) for one or more aeroallergens.

Patients were subsidiary to receive subcutaneous AIT (in any composition, formulation, or administration schedule) according to clinical recommendations, and thus including both monosensitized and polysensitized patients.

Pregnant or lactating women were not eligible, as they were not susceptible to receive treatment with immunotherapy, according to the usual clinical practice following EAACI recommendations (15). All patients were evaluated at baseline, and were recalled after 6 months of treatment for a follow-up visit, and after 1 year of initiating AIT treatment for the final visit evaluation performance.

\section{Outcomes measures}

Quality of Life. To assess the changes in patients' QoL after treatment, Rhinoconjunctivitis Quality of Life Questionnaire (RQLQ) developed and validated by Juniper $(26,27)$ was used. The questionnaire consists on a self-administered version validated in Spanish for patients over 12 years. RQLQ involves 28 items-questions distributed in 7 domains (activities 3 items, sleep disturbances 3 items, general problems 7 items, nose symptoms 4 items, eye symptoms 4 items, and emotional function 4 items). Responses are scored on a 7-point Likert scale, while domains and overall score are scored on a 0-to-6 scale $(0=$ not troubled; $6=$ extremely troubled $)$, with lower scores indicating better QoL. Comparisons were made between the mean scores of the RQLQ obtained at baseline visit, and at the one-year post-treatment visit. As previously reported, a change greater than 0.5 on the RQLQ domain and overall scores is the critically meaningful "minimal important difference" (MID) or clinically significant difference $(22,28)$.

Impact on AR and Asthma. The mean number of AR episodes suffered by patients within the last year before AIT treatment initiation, and after 1-year receiving treatment was described and compared between. On the other hand, the classification of AR, ARIA (3), was used to assess the clinical status of patients, before and after treatment (type and intensity). The classification of allergic asthma (GINA) (29) was also used to assess the presence or absence of asthmatic symptoms in patients, before and after AIT treatment. The intake of symptomatic medication before the start of treatment, as well as after 1-year SCIT treatment was also evaluated.

Adherence to AIT treatment. The percentage of patient's therapeutic compliance with treatment was evaluated. Patients who had completed at least $80 \%$ of established doses were considered to be good compliant.

Satisfaction with AIT treatment. After treatment, patients' and physician's satisfaction with received AIT was assessed using a visual analogue scale (VAS) from each point of view. This scale ranges from 0 to 10 , being 10 the highest degree of imaginable satisfaction, and 0 the lowest degree of satisfaction that may exist. Factors associated with AIT efficacy. Through a multivariate analysis, the identification of the possible patient and treatment associated factors that may had influenced in the patients' QoL changes after treatment, was analyzed through the assessment of the following variables:

- patient's age (children / adults);

- patient's sex (male / female);

- therapeutic compliance (good compliant / non-compliant);

- level of studies (without studies / primary studies / professional training / high school / higher technical degree / higher degree);

- socioeconomic level (very low / low / medium- low / medium / medium-high / high);

- type of center (public/ private);

- degree of physician satisfaction with AIT ( $<$ or $\geq$ of the median);

- degree of patient satisfaction with AIT $(<$ or $\geq$ of the median);

- prescribed AIT treatment: source (pollens / mites / others); composition (single source / mixture of extracts); schedule (cluster / fast / conventional / other); formulation (depot / polymerized);

Safety. For safety assessment, any adverse reaction occurred during treatment and detected either by patient or by physician was recorded. A patient's diary was used for these purposes.

\section{Statistical analysis}

Safety and descriptive analyses were performed using the safety population (receiving at least one dose). Efficacy statistical analyses were performed using the intention-to-treat, (ITT) population. The categorical variables were described by absolute and relative frequencies. For the description of the continuous variables, mean and standard deviation were used. For the comparison of the quantitative variables of two or more independent groups, parametric tests (Student's t test or ANOVA) or non-parametric tests (U of Mann-Whitney or Kruskal-Wallis) 
were used. For the comparison of two or more paired groups parametric tests (Student's t-test for paired data or analysis of the variance of repeated measures) or non-parametric tests (Wilcoxon or Friedman), were used, according to the characteristics of the variables under study (normality) and the number of groups to compare. For the qualitative variables, the Chisquare test or Fisher's exact test was used to compare patients' subgroups, or either McNemar test or Bhapkar test (table KxK, $\mathrm{k}>2$ ) for comparisons between visits. Correlations (Pearson or Spearman's rho) were used to study the relationship between 2 quantitative variables. In all statistical tests, a bilateral statistical significance level of 0.05 was applied.

To study the possible associated variables of influence on patients' QoL through a multivariate analysis, a multiple linear regression was performed using the "backward" procedure with an exit probability of 0.10 . All the statistical analysis of the data was carried out with the support of the statistical package SAS version 9.4 .

\section{Results}

\section{Descriptive data}

A total of 127 patients from 13 Allergy Departments were recruited, and 120 of them could be included and analyzed (7 patients did not start AIT treatment). All study sites were distributed in different regions of the inland area of Spain, except one site that was in the coastal area. Patients' mean age was 32.93 years $(S D=13.2), 22$ of them were under 18 years old $(18.3 \%)$. $45.8 \%$ of patients were men. $77.5 \%$ of patients expressed a socioeconomic level classified as medium and high, and a $21.7 \%$ as low or medium-low. Regarding levels of education-formation, $35.7 \%$ of patients (or tutors) had a bachelor's degree, as the most frequent one (table I). Forty out of the 120 patients (33.3\%) had allergy family background. According to ARIA classification (3), most patients were classified as persistent AR (82.5\%) and moderate / severe intensity $(80.0 \%)$, being concomitantly persistent and moderate / severe in the $67.5 \%$ of all cases (table II). Following the criteria of GINA guidelines (29), 66.7\% of patients presented associated asthma at baseline, being 95.5\% of them mild asthma cases. The mean number of episodes of
AR/year suffered by patients at baseline was $18.59(\mathrm{SD}=28.4)$ episodes. At baseline, almost half of the patients (48.1\%) were previously on symptomatic treatment, $82.76 \%$ of them with antihistaminic drugs, and $55.17 \%$ with nasal corticosteroids. Regarding subcutaneous AIT composition, $60.0 \%$ of treatments contained a single allergenic source, while the remaining $40.0 \%$ contained some extracts mixture. $52.9 \%$ of cases were in native depot, and $47.1 \%$ in polymerized formulation (table I). Abbreviated conventional administration schedule was the most prescribed $(70.0 \%)$, followed by clustered one $(25.0 \%)$. AIT treatments contained some type of pollen in its composition in $92.5 \%$ of cases, either as unique source $(56.8 \%)$ or as any

\section{Table I - Patients' demographic and baseline characteristics.}

\begin{tabular}{ll}
\hline Baseline characteristics & $\mathbf{n}=\mathbf{1 2 0}$ \\
\hline Age (years), mean (SD) & $32.93(13.21)$ \\
\hline Age categories, $\mathrm{n}(\%)$ & $22(18.3)$ \\
12-17 years & $98(81.7)$ \\
$\geq 18$ years & \\
\hline Gender, $\mathrm{n}(\%)$ & $55(45.8)$ \\
men & $65(54.2)$ \\
women & \\
\hline Race, $\mathrm{n}(\%)$ & $102(85.0)$ \\
Caucasian & $1(0.8)$ \\
Sub-saharan & $16(13.3)$ \\
Iberoamerican & $1(0.8)$ \\
Asiatic & $80(66.7)$ \\
\hline Concomitant asthma, $\mathrm{n}(\%)$ & \\
\hline Extract type, $\mathrm{n}(\%)$ & $111(92.5)$ \\
pollen & $6(5.0)$ \\
mites & $3(2.5)$ \\
others & \\
\hline Extracts source type, $\mathrm{n}(\%)$ & $72(60.0)$ \\
unique & $48(40.0)$ \\
mixtures & $56(47.1)$ \\
\hline SCIT formulation $\mathrm{n}(\%)$ & \\
polimerized & \\
native depot & \\
\hline
\end{tabular}

Table II - Patients' AR classification in frequencies and percentages (ARIA)

\begin{tabular}{ccccc}
\hline \multirow{2}{*}{ Type } & \multicolumn{3}{c}{ Intensity } & \multicolumn{2}{c}{ Total } \\
& & mild & moderate / severe & $21(17.5 \%)$ \\
\cline { 2 - 5 } & intermittent & $6(5.0 \%)$ & $15(12.5 \%)$ & $99(82.5 \%)$ \\
\cline { 2 - 5 } & persistent & $18(15.0 \%)$ & $81(67.5 \%)$ & $120(100 \%)$ \\
\hline
\end{tabular}


pollen's mixture (43.2\%). An additional 5\% contained mites (single source or mixtures), and $2.5 \%$ other extracts, reflecting that almost all patients belonged to inland areas of Spain. Grass pollen as unique source $(68.1 \%$ of the total number of unique treatments) and the combination of grass pollen with Olea europaea pollen (43.8\% of the combined mixtures treatments) were the most frequently prescribed compositions.

\section{Quality of Life (RQLQ)}

One hundred and three patients completed the study providing primary outcome data. Total score means values in RQLQ questionnaire decreased from 2.61 to 1.34 points in final visit (oneyear treatment), reflecting a clinically and statistically significant improvement ( $\mathrm{p}<0.01$, Wilcoxon test). The absolute change in score is clearly above the established MID (0.5 points) in RQLQ values and, therefore, represents a clinically significant difference for patients. These values of 1.27 points in absolute change, constitute a mean value of $32.4 \%(\mathrm{SD}=80.59)$ in relative change between basal-final study visits (figure 1). Complete data are described in table III. There were clinically and statistically significant reductions in all of the 7 different domains that constitute the RQLQ questionnaire. The domain with the greatest improvement in absolute change was the eye symptoms, with 1.50 points, $41.5 \%$ of relative change from baseline ( $\mathrm{p}<$ 0.01 ), followed by sleeping ( 1.44 points, $56.0 \%$ relative change) and practical problems (1.41 points, $33.9 \%$ relative change). Every remaining domain improved in more than 1.0 point in absolute change and at least in $20 \%$ in relative change.

\section{Impact on AR and asthma}

In addition, the average number of $A R$ annual episodes decreased from $18.77(S D=29.31)$ to $8.75(S D=30.39)$ after one-year AIT treatment, being a statistically significant difference $(\mathrm{p}<$ 0.01 , Wilcoxon test) and representing a $46.6 \%$ of reduction. The $43.7 \%$ of patients improved after 1-year post-treatment from persistent to intermittent AR ( $\mathrm{p}<0.01)$, and $40.8 \%$ from moderate/severe to mild intensity (ARIA) $(p<0.01)$ (table IV). Significantly, a $29.1 \%$ of patients improved from persistent and moderate-severe AR to an intermittent and mild AR. An additional $15.5 \%$ of the former patients improved at the end of the follow up, being categorized in lower grades according to the basal gradation. Regarding the classification of allergic asthma (GINA), $17.5 \%$ of asthmatic patients at baseline, did not have any bronchial symptoms after 1-year AIT treatment (table V) (p

Figure 1 - Mean relative changes (\%) in RQLQ global score and different RQLQ items between baseline and final visits.

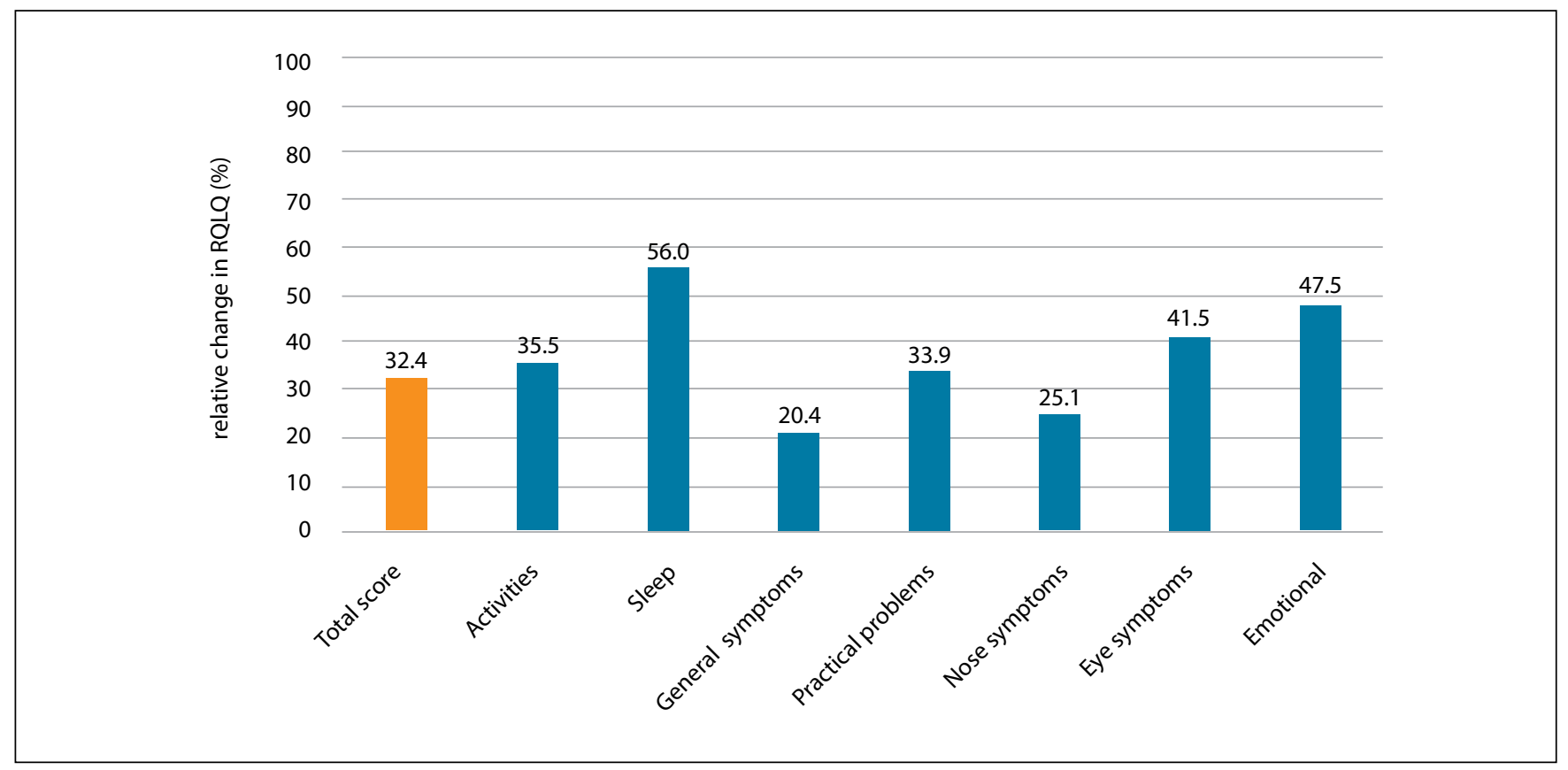

Relative change between visits: ([value of baseline visit - value one-year visit] / value of baseline visit) $\mathrm{x} 100$; percentages higher than 0 indicate improvement in the QoL. Note. Calculated only in patients with baseline values $>0$. 
Table III - Evolution of the RQLQ scores after one-year AIT treatment.

\begin{tabular}{|c|c|c|c|c|}
\hline & Baseline visit & One-year visit & Absolute change $^{1}$ & $\mathbf{p}^{2}$ \\
\hline \multicolumn{5}{|c|}{ RQLQ total score } \\
\hline mean $(\mathrm{SD})$ & $2.61(1.58)$ & $1.34(1.21)$ & $1.27(1.64)$ & $<0.0001$ \\
\hline median & 2.96 & 0.89 & 1.43 & \\
\hline \multicolumn{5}{|l|}{ Activities } \\
\hline $95 \% \mathrm{CI}$ & $(2.46-3.15)$ & $(1.21-1.81)$ & $(0.89-1.70)$ & \\
\hline median & 3.00 & 1.00 & 1.67 & \\
\hline \multicolumn{5}{|l|}{ Sleep } \\
\hline mean $(\mathrm{SD})$ & $2.37(1.98)$ & $0.93(1.38)$ & $1.44(2.05)$ & $<0.0001$ \\
\hline mean $(\mathrm{SD})$ & $2.41(1.62)$ & $1.35(1.23)$ & $1.06(1.57)$ & $<0.0001$ \\
\hline $95 \%$ CI & $(2.10-2.73)$ & $(1.11-1.59)$ & $(0.76-1.37)$ & \\
\hline median & 2.57 & 1.00 & 0.86 & \\
\hline \multicolumn{5}{|c|}{ Practical problems } \\
\hline mean $(\mathrm{SD})$ & $3.24(1.96)$ & $1.83(1.68)$ & $1.41(2.11)$ & $<0.0001$ \\
\hline $95 \% \mathrm{CI}$ & $(2.86-3.62)$ & $(1.50-2.16)$ & $(1.00-1.82)$ & \\
\hline median & 3.67 & 1.33 & 1.00 & \\
\hline \multicolumn{5}{|c|}{ Nose symptoms } \\
\hline median & 2.75 & 0.75 & $1-25$ & \\
\hline \multicolumn{5}{|l|}{ Emotional } \\
\hline mean $(\mathrm{SD})$ & $1.82(1.57)$ & $0.80(1.07)$ & $1.02(1.52)$ & $<0.0001$ \\
\hline $95 \% \mathrm{CI}$ & $(1.51-2.12)$ & $(0.59-1.01)$ & $(0.72-1.31)$ & \\
\hline median & 1.75 & 0.25 & 0.75 & \\
\hline
\end{tabular}

Note. Low scores in the RQLQ questionnaire indicate a better QoL (scale 0-6). ${ }^{1}$ Absolute change between visits: (value of baseline visit - value of one-year visit). ${ }^{2}$ Wilcoxon test

$<0.01$, McNemar test). Moreover, none of the patients without asthma symptoms at basal visit developed bronchial symptoms at the end of one-year treatment.

In the case of patients who had previously taken antihistamine medications, $68.5 \%$ of them (26 of 38 patients) decreased or stopped their intake after 1-year treatment with SCIT. Only 1 patient increased its use. In the case of patients taking nasal corticosteroids, $67.8 \%$ of them (26 of 28 patients) decreased or stopped their use after 1 -year treatment. Only 2 patients increased their use. 
Table IV - AR classification (ARIA) evolution after one-year AIT treatment.

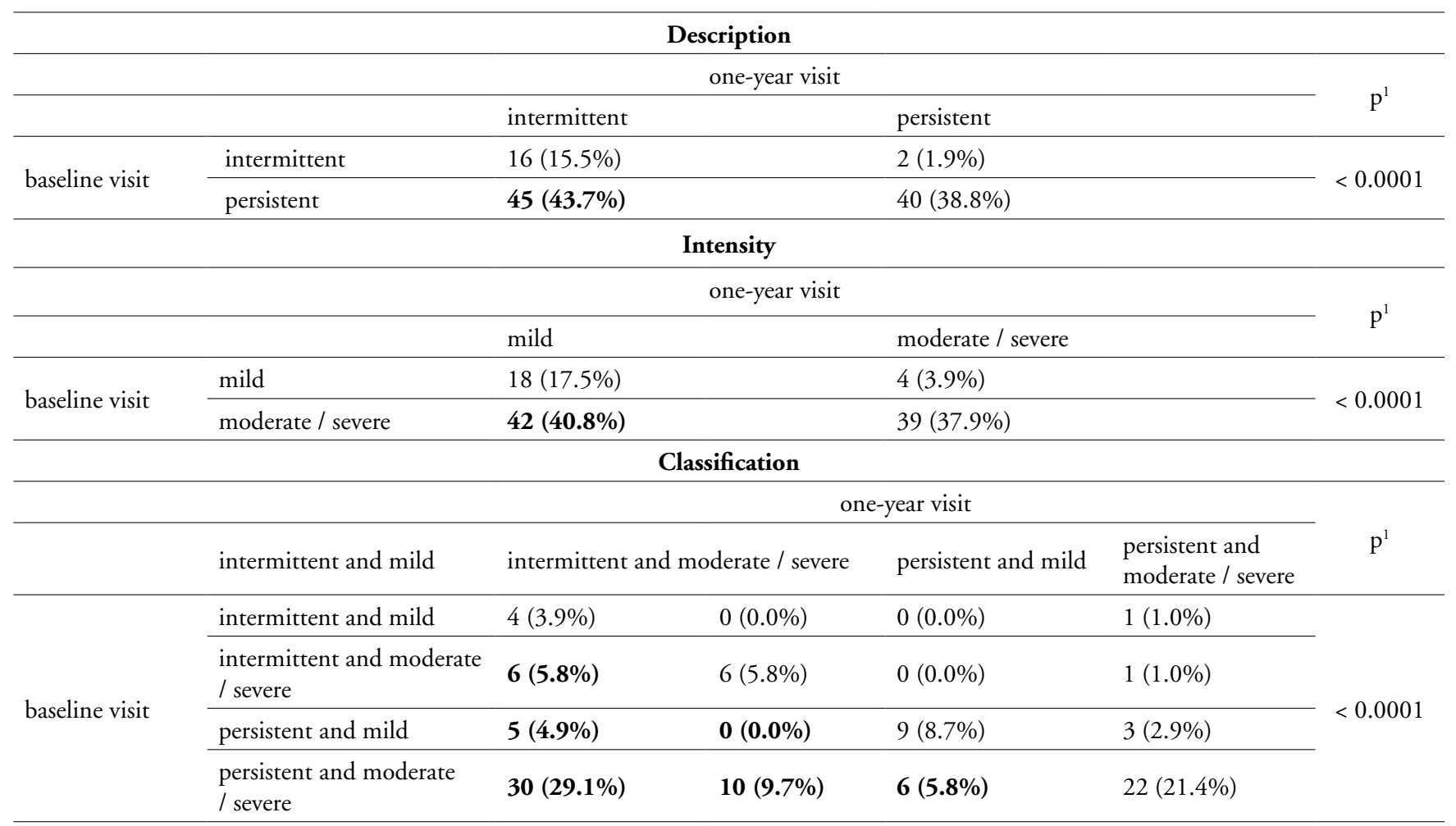

$\mathrm{n}(\%)$ : (\% calculated with $\mathrm{n}=103) .{ }^{1}$ Bhapkar test (table KxK, $\left.\mathrm{k}>2\right)$.

Table $\boldsymbol{V}$ - Evolution of the of allergic asthma classification (GINA) after one-year AIT treatment.

\begin{tabular}{|c|c|c|c|c|}
\hline \multicolumn{5}{|c|}{ Asthma presence } \\
\hline & & \multicolumn{2}{|c|}{ one-year visit } & \multirow[t]{2}{*}{$\mathrm{p}^{1}$} \\
\hline & & yes & no & \\
\hline \multirow[t]{2}{*}{ baseline visit } & yes & $51(49.5 \%)$ & $18(17.5 \%)$ & $<0.0001$ \\
\hline & no & $0(0 \%)$ & $34(33 \%)$ & \\
\hline
\end{tabular}

${ }^{1}$ McNemar Test.

\section{Adherence}

Regarding patients' adherence with AIT treatment, mean percentage of therapeutic compliance in SCIT was $92.0 \%$ of patients (patient who completed at least $80 \%$ of established doses). When comparing compliance between different treatment types, a minor compliance was seen in patients treated with allergen mixtures $(85.0 \%$ for mixtures vs $95.1 \%$ for unique source treatments), whereas no statistically significant difference was detected. No differences were also detected between pollen vs mites AIT treated patients ( $\mathrm{p}>0.05$ Fisher test).

\section{Satisfaction}

After one-year AIT treatment, patients' and physician's satisfaction was assessed using a Visual Analogue Scale (VAS) from both perspectives. Mean values of treatment satisfaction were 7.24 $(\mathrm{SD}=1.90)$ and $7.05(\mathrm{SD}=1.83)$ for patients and physicians respectively. A clear correlation was observed between both values obtained by patients and physicians ( $p<0.1$, Rho Spearman).

\section{Factors associated with AIT efficacy}

The pre-requisite for analyzed variables to enter in the multiple linear regression model, was to obtain a p-value lower to 0.10 in the univariate analysis. The variables that complied and were included in the multivariate analysis were the following:

- degree of physician satisfaction with AIT;

- degree of patient satisfaction with AIT;

- composition (single source / mixture of extracts);

- formulation (depot / polymerized).

The introduced variables were eliminated one by one by the "backward" method, with an exit probability of 0.10 . Only the variable "patient satisfaction" remained in the multivariate re- 
gression model $(\mathrm{p}<0.01)$, meaning that after treatment, patients who reported greater satisfaction values with the treatment, also improved significantly more in their QoL, than those with lower satisfaction values.

\section{Safety assessment}

The safety analysis was performed considering the total number of patients recruited in the study, with data from a follow-up visit (safety population, $\mathrm{n}=120$ patients who had at least some information during the 6-month visit). Of the 238 adverse events recorded, 233 were definitely, probably or possibly related to the study medication, therefore were considered adverse reactions. Thus, 54 patients (45.0\%) had a total of 233 adverse reactions. None of the adverse reactions was serious, being the majority of them $(90.8 \%)$ classified as mild, thus only $9.2 \%$ were of moderate intensity. $69.1 \%$ of the adverse reactions were registered within 0-6 months period, while the rest (30.9\%) within the $6-12$ months treatment period. $54.3 \%$ of the adverse reactions occurred in the treatment initiation phase, and the rest $(45.7 \%)$ in the maintenance phase.

Local reactions in the injection area accounted for $89.7 \%$ of local reactions (209 reactions) being $62.2 \%$ of them delayed. Only $6.2 \%$ of these local reactions were clinically relevant (classified as moderate or severe intensity).

Only 24 systemic reactions were reported in 11 patients $(9.17 \%$ of patients). Of them, 18 were of grade I (in 9 patients), and 6 of grade II (in 2 patients). One patient suffered a total of 5 grade II reactions, due to a dosage error in primary care, consisting in generalized itching, muscle pain, rhinitis and shortness of breath during 24-48 hours. The other patient with a grade II reaction, presented general discomfort, respiratory distress and ocular itching 48 hours after the AIT administration. Thus, only the $1.67 \%$ of patients suffered grade II reactions. There were no systemic reactions of grades higher than II (EAACI grading system) (table VI).

Taking into account the total number of doses administered (1712 doses), adverse reactions accounted for the $13.6 \%$ of them, being systemic reactions on the $1.4 \%$ of doses. Only the $1.2 \%$ of doses caused some moderate intensity adverse reactions ( $0.8 \%$ local and $0.4 \%$ systemic reactions).

\section{Discussion}

The efficacy and safety of immunotherapy has been very well documented in multiple well-designed and controlled clinical trials. Patients in these studies are usually very selected and rigorously controlled, which does not happen in daily clinical practice. This study evaluated the behaviour of immunotherapy in real life conditions, giving an idea of the profile of patients receiving immunotherapy and the type of treatment prescribed. The results of this study confirmed not only the improvement in the QoL, but also the impact on the symptoms of rhinitis and asthma, the decrease in medication, the good adherence to treatment, and the safety of AIT.

AIT is the only treatment option that can induce specific immune tolerance and has long-term disease-modifying effect, inducing desensitization $(9,15,16,17,18)$. Several validated tools for assessing HRQoL in AR are currently available (30). The most frequently used specific and validated instrument, involved in AIT trials is the RQLQ questionnaire $(23,26,27)$. Moreover, only the RQLQ allows calculating the MID, namely how much a score must change so that it is perceived as such by the patient, irrespective of its statistical significance (31).

Table VI - Adverse reactions classification and description.

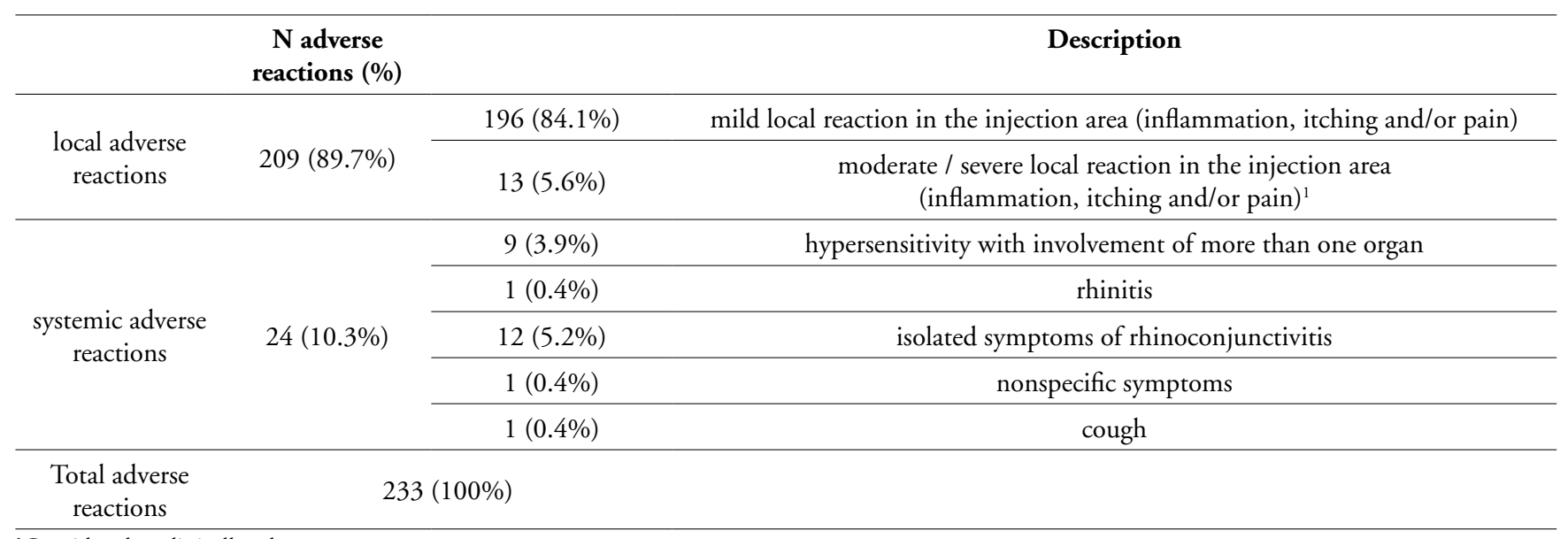

\footnotetext{
${ }^{1}$ Considered as clinically relevant.
} 
In this study, a clear and important improvement in patients' HRQoL was observed compared to baseline, both globally in the total score, and in each of the different domains that form the validated and disease specific RQLQ questionnaire, after one-year AIT treatment. All observed improvements in RQLQ were clearly above the threshold of 0.5 points of change, for a clinically important improvement, previously defined by Juniper $(31,32)$. Interestingly, the domain in which patients improved the most in absolute change, was the related to eye symptoms. On the other hand, there were also significant improvements in the number of AR episodes suffered by patients per year, as well as in the type and intensity of their pathology, according to the ARIA classification. In addition, focusing in patients with associated asthma at baseline, a significant percentage of them, did not present any bronchial symptoms after AIT treatment. Although with minor differences depending on the composition (mixtures vs single source), the patients' compliance with the treatment was very high, probably because subcutaneous AIT requires to be administered by a healthcare professional. This is important, since adherence to AIT in real-life, especially to the recommended prolonged courses, could be an issue and compromise the efficacy demonstrated in clinical trials. At the end of the study, both patients and physicians reported high and correlated satisfaction values with the treatment in VAS score, in concordance with the positive results observed also in patient's QoL improvement, and AR and asthma positive impact.

Limitations of this study are those of a non-interventional prospective, uncontrolled study in the real-life setting, like unpredictable bias, confusion bias and selection bias. In order to minimize a potential investigator and selection bias of the study, sites distributed all over Spain were involved. Moreover, given that in the study the different demographic, clinical and treatment factors that may had an influence on efficacy were analyzed, we consider that the possibility of confusion bias is reduced when interpreting these results.

On the other hand, treatments that contained pollen from grasses were the most widely received by the patients (either alone or in combination with, Olea or other extracts), involving $85.4 \%$ of total patients' treatments. Regarding a possible influence on the positive observed results, of a lower pollen counts in the spring in which patients were in treatment (2016), in comparison with the pollen counts in the spring before treatments started (2015), it must be clarified that grasses pollen counts in Spain in 2016 were much higher than in the previous year 2015. Given these facts, it is not possible to assign the improvement in patients' QoL to a lower level of pollens during the year receiving the treatments.

Whereas in the past traditional clinical measures were supposed to provide a comprehensive description of the impact of the disease on patients, it is now proved that HRQoL is a necessary parameter for achieving a more complete assessment of allergic diseases. In long-term treatments like AIT, improvements of the disease-specific HRQoL are especially important.

Exploring the improvements in absolute change values in the different domains of the RQLQ, the eye symptoms had the greatest change observed (1.50 points), followed by sleep (1.44 points) and practical problems (1.41 points). Eye symptoms is the aspect that, together with nasal symptoms, has been found to strongly affect HRQoL $(33,8)$. Eye symptoms have a significant impact on daily activities and work or school performance. At the same time, they are some of the most difficult to control (23). These results go in accordance with those reported by Novakova et al recently (23). By the other hand, AR is known to affect nocturnal sleep and daytime sleepiness which may be related to nasal congestion (34). Additionally, lack of sleep has consequences for both social functioning and school performance (35). Given all of the above, sleep disturbances related to AR have clear significant implications on HRQoL (35).

By the other hand, as a result of their symptoms patients with AR run into daily practical problems such as the discomfort to carry tissues, the need to rub their nose / eyes, and have to blow their nose many times. These problems could potentially interfere with their social interaction, limiting their activities. The improvement of these "practical problems" such as that observed in this study, undoubtedly contributed to the overall HRQoL improvement as a result of AIT treatment.

Also, significant improvements were observed in all the rest of the domains like "activities", general symptoms, nose symptoms and emotional aspects after one-year of treatment, contributing, to the global improvement shown of patients' HRQoL. Maybe the impact of AIT on all these aspects might explain at the same time the improvement of their emotional wellbeing.

The positive changes in RQLQ observed in this study, are in the same line that those previously reported in some randomized clinical trials where this effect has been evaluated for AIT $(21,22,36,37)$. Nowadays, few observational studies, under clinical routine conditions, have shown the benefits of AIT treatment in improving the QoL of patients with $\operatorname{AR}(38,39)$. Interestingly, Schwanke et al. (40) in 2017 published a observational study with the objective to compare changes in QoL with sublingual immunotherapy (SLIT) and SCIT treatments in patients with AR in a real-world clinical setting. They concluded that although improvements in QoL were noted in both groups, changes in overall scores and the majority of domains only achieved statistical significance in the SCIT group. Some other non-interventional studies have also evaluated improvements in QoL in patients with AR, focusing only in SLIT treatments, and showing also positive results $(23,24,41)$. Interestingly, in the study published by Horn (24), routine treatment with a grass SLIT-tablet resulted in clear improvements in disease-specific and general quality of life, while no 
improvements were observed in patients treated only symptomatically.

Among the findings of this study, it is important to note the importance of seeing a significant reduction in the percentage of patients with associated asthma who showed bronchial symptoms after the year of treatment with AIT. As stated in the last GINA Report, in people with asthma and allergic sensitization, SCIT is associated with a reduction in symptom score and medication requirements, and improved allergen specific and non-specific airway hyper responsiveness (42). These findings go in accordance with the mentioned in last published GINA report, and other systematic reviews analyzing the benefits of AIT in asthma (43).

As part of the secondary objectives of this study, we explored the possible influence of some patient and treatment factors on the efficacy of treatment, regarding the improvement of HRQoL as the primary study endpoint. However, only "degree of patient satisfaction" appeared to be independent clinical predictor when multiple factors were accounted for, in the predictive model, as might be expected. On the other hand, it is worth noting that in this study, no significant differences were detected between the subgroups of analyzed patients, regarding demographic factors such as age (children vs. adults) or sex (male vs. female), with respect to the efficacy of treatment in patients' QoL improvement. Few studies evaluated the impact of the therapies on children and adolescent suffering from AR. Our findings in this regard are in accordance with those reported by Filanowicz et al. in 2016 (44), where no significant correlation between sex and age of examined people and the improvement of QoL was found, in patients with AR after AIT. No differences were neither found regarding the improvement in the QoL associated with the patients' socioeconomic characteristics.

Another aspect to be considered is the safety and tolerability of AIT treatment showed in this observational study. It can be affirmed that the safety profile is good, given that few systemic reactions associated with the treatment were reported, and at the same time few of them were of moderate intensity and in a small number of patients. The vast majority of adverse reactions reported were local, at the injection site, and consisted of erythema, inflammation, pain, and/or swelling, being only the $6.2 \%$ of them clinically relevant.

An important fact is that this study included different types of treatment composition (pollens and mites) sources of allergens (single and mixtures) as well as different formulations (polymerized / depot) not observing differences associated with the improvement of QoL, for the different subgroups analyzed.

The data observed in this study can be useful for physicians' decision-making when managing patients with AR, regarding whether a patient could be benefit from AIT treatment.

\section{Conclusions}

The results of this study provide evidence that a one-year treatment with subcutaneous AIT (ROXALL Medicina España S.A.), significantly increases $\mathrm{QoL}$ in patients with $\mathrm{AR}$, together with a significant positive impact on AR type and intensity, and a reduction in the percentage of patients showing asthmatic symptoms after treatment.

Moreover, high patients' satisfaction values with treatment were reported, together with an adequate safety profile with a low number of systemic adverse reactions, none of them serious.

\section{Acknowledgements}

The authors would like to thank the inestimable collaboration of the rest of the principal investigators participating in the study sites which are the following: Javier Hdez. Arbeiza, Eloína González, Gabriela Zambrano, Jesus Trujillo, Mónica Rodríguez, Antonia Padial, Raquel López, Carlos Colás, Alicia Alonso. The authors would like to thank also to all the remaining collaborating investigators who had participated in each of the study sites, and to Manuel de las Heras, Javier Dionicio, Rosario Gonzalez, Aranzazu Jimenez, Esther Compes and Felicitas Villas, as well as the collaboration of Nerea González, in the development of this study.

\section{Funding}

This study was funded by ROXALL Medicina España S.A.

\section{Conflict of interest}

LB, BM, MCG and AM belong to the R and D department of ROXALL Medicina España S.A, the company sponsor of this study.

\section{References}

1. Greiner AN, Hellings PW, Rotiroti G, Scadding GK. Allergic rhinitis. Lancet 2011; 378(9809):2112-2122.

2. Skoner DP. Allergic rhinitis: Definition, epidemiology, pathophysiology, detection, and diagnosis. J Allergy Clin Immunol 2001; 108(1 Suppl):S2-8.

3. Brozek JL, Bousquet J, Baena-Cagnani CE, Bonini S, Canonica GW, Casale TB, van Wijk RG, Ohta K ZT. Allergic Rhinitis and its Impact on Asthma (ARIA) guidelines: 2010 Revision. J Allergy Clin Immunol 2010; 126(3):466-476.

4. Rabe KF, Vermeire PA, Soriano JB, Maier WC. Clinical management of asthma in 1999: The Asthma Insights and Reality in Europe (AIRE) study. Eur Respir J 2000; 16(5):802-807.

5. Sociedad Española de Alergología e Inmunología Clínica (SEAIC). Alergológica 2015. Factores epidemiológicos, clínicos y socioeconómicos de las enfermedades alérgicas en España en 2015. SEAIC 2017; 2015. 
6. Mallol J, Crane J, Mutius E Von, Odhiambo J, Keil U, Stewart A. The International Study of Asthma and Allergies in Childhood (ISAAC) Phase Three: A global synthesis. Allergol Immunopathol 2013; 41 (2):73-85.

7. Patil VK, Kurukulaaratchy RJ, Venter C, Grundy J, Roberts G, Dean T, Arshad SH. Changing prevalence of wheeze, rhinitis and allergic sensitisation in late childhood: findings from 2 Isle of Wight birth cohorts 12 years apart Experimental Allergy 2015; 45 (9):1430-1438.

8. Bousquet PJ, Demoly P, Mesbah K. Impact of Allergic Rhinitis Symptoms on Quality of Life in Primary Care. Int Arch Allergy Immunol 2013; 160(4):393-400.

9. Dhami S, Nurmatov U, Arasi S, Khan T, Asaria M, Zaman H. Allergen immunotherapy for allergic rhinoconjunctivitis: A systematic review and meta-analysis. Allergy 2017; 72(11):1597-1631.

10. Guerra S, Sherrill DL, Martinez FD, Barbee RA. Asthma, rhinitis, other respiratory diseases rhinitis as an independent risk factor for adult-onset asthma. J Allergy Clin Immunol 2002; 109(3):419-425.

11. Canonica GW, Mullol J, Didier A. Patient Perceptions of Allergic Rhinitis and Quality of Life Findings from a Survey Conducted in Europe and the United States. World Allergy Organ J 2008; 1(9):138-144.

12. Thompson AK, Juniper E, Meltzer EO. Quality of life in patients with allergic rhinitis. Ann Allergy, Asthma Immunol 2000; 85(5):338-347.

13. Bachert C, Bousquet J, Canonica GW, Durham R, Klimek L, Mullol J. Levocetirizine improves quality of life and reduces costs in long-term management of persistent allergic rhinitis. J Allergy Clin Immunol 2004; 114(4):838-844.

14. Zuberbier T, Bachert C, Bousquet PJ, Passalacqua G, Walter Canonica G, Merk H et al. GA 2 LEN / EAACI pocket guide for allergen-specific immunotherapy for allergic rhinitis and asthma. Allergy 2010; 65(12):1525-1530.

15. Roberts G, Pfaar O, Akdis CA, Ansotegui IJ Durham SR Gerth van Wijk R et al. EAACI Guidelines on Allergen Immunotherapy: Allergic rhinoconjunctivitis. Allergy 2018; 73(4):765-798.

16. Nurmatov U, Dhami S, Arasi S, Roberts G, Pfaar O, Muraro A et al. Allergen immunotherapy for allergic rhinoconjunctivitis: a systematic overview of systematic reviews. Clin Transl Allergy 2017; 8:7:24

17. Jutel M, Agache I, Bonini S, Burks AW, Calderon M, Canonica W, et al. International Consensus on Allergen Immunotherapy II: Mechanisms, standardization, and pharmacoeconomics. J Allergy Clin Immunol 2016; 137(2):358-368.

18. Alvarez-Cuesta E, Bousquet J, Canonica GW, Durham SR, Malling H, Valovirta E. Standards for practical allergen-specific immunotherapy. Allergy 2006; 61(Suppl 82):1-3.

19. Baiardini I, Braido F, Brandi S, Canonica GW. Allergic diseases and their impact on quality of life. Ann Allergy Ashtma Immunol 2006; 97(4):419-429.

20. Carlos C, Monzo S, Venturini M, Lezaun A. Double-blind, placebo-controlled study with a modified therapeutic vaccine of Salsola kali (Russian thistle) administered through use of a cluster schedule. J Allergy Clin Immunol 2006; 117(4):810-816.

21. Frølund L, Durham SR, Calderon M, Emminger W, Andersen JS, Rask P et al. Sustained effect of SQ-standardized grass allergy immuno-therapy tablet on rhinoconjunctivitis quality of life. Allergy 2010; 65(6):753-757.
22. E. Alvarez-Cuesta, E. Aragoneses-Gilsanz, C. Martın-Garcia, Berges-Gimeno P, E. Gonzalez-Mancebo, J. Cuesta-Herranz. Immunotherapy with depigmented glutaraldehyde-polymerized extracts: changes in quality of life. Clin Exp Allergy 2005; 35(5):572-578.

23. Novakova SM, Staevska MT, Novakova PI, Yoncheva MD, Bratoycheva MS, Musurlieva NM. Quality of life improvement after a three- year course of sublingual immunotherapy in patients with house dust mite and grass pollen induced allergic rhinitis: results from real-life. Health Qual Life Outcomes 2017; 29;15(1):189

24. Horn A, Zeuner H, Wolf H, Schnitker J. Health-Related Quality of Life During Routine Treatment with the SQ-Standardised Grass Allergy Immunotherapy Tablet: A Non-Interventional Observational Study. Clin Drug Investig 2016; 36(6):453-462.

25. Elm E Von, Altman DG, Egger M, et al. The Strengthening the Reporting of Observational Studies in Epidemiology (STROBE) Statement: Guidelines for reporting observational studies. Int J Surg 2014; 12(12):1495-1499.

26. Juniper EF, Guyatt GH. Development and testing of a new measure of health status for clinical trials in rhinoconjunctivitis. Clin Exp Allergy 1991; 21(1):77-83.

27. Juniper EF, Thompson AK, Ferrie PJ, Roberts JN, City K. Validation of the standardized version of the Rhinoconjunctivitis Quality of Life Questionnaire. J Allergy Clin Immunol 1999; $104(2$ Pt 1):364-369.

28. Juniper EF1, Guyatt GH, Willan A, Griffith LE. Determining a minimal important change in a disease-specific Quality of Life Questionnaire. J Clin Epidemiol 1994; 47(1):81-87.

29. GINA Report: Global Strategy for Asthma Management and Prevention, Global Initiative for Asthma (GINA) 2015; https:// ginasthma.org 2015.

30. Braido F1, Bousquet PJ, Brzoza Z, Canonica GW, Compalati E, Fiocchi A, et al. Specific recommendations for PROs and HRQoL assessment in allergic rhinitis and/or asthma: a GA²LEN taskforce position paper. Allergy 2010; 65(8):959-968.

31. Juniper EF. Quality of life questionnaires: Does statistically significant = clinically important? J Allergy Clin immunol 1998; 102(1):16-17.

32. Juniper EF, Guyatt GH, Griffith LE, Ferrie PJ. Interpretation of rhinoconjunctivitis quality of life questionnaire data. J Allergy Clin Immunol 1996; 98(4):843-845.

33. G C, Klersy C, Cirillo I, Marseglia GL. Quality of life in allergic rhinitis: relationship with clinical, immunological, and functional aspects Clinical and Experimental Allergy. Clin Exp Allergy 2007; 37(10):1528-1535.

34. Meltzer EO. Introduction: Stuffy is also related to Sleepy and Grumpy - The link between rhinitis and sleep-disordered breathing. J Allergy Clin Immunol 2004; 114 (5 Suppl):S133-134.

35. Baiardini I, Braido F, Cauglia S, Canonica GW. Sleep disturbances in allergic diseases. Allergy 2006; 61(11):1259-1267.

36. Walker SM, Pajno GB, Lima T, Wilson DR, Durham SR, Kingdom U. Grass pollen immunotherapy for seasonal rhinitis and asthma: A randomized, controlled trial. J Allergy Clin Immunol 2001; 107(1):87-93.

37. Nelson HS, Nolte H, Creticos P, et al. Efficacy and safety of timothy grass allergy immunotherapy tablet treatment in North American adults. J Allergy Clin Immunol 2011; 127(1):72-80.

38. Petersen KD, Kronborg C, Larsen JN, Dahl R, Gyrd-Hansen D. Patient related outcomes in a real life prospective follow up study: 
Allergen immunotherapy increase quality of life and reduce sick days. World Allergy Organ J 2013; 6(1):15.

39. Mahler V, Klein C, Sager A. House dust mite-specific immunotherapy with two licensed vaccines: Outcome under clinical routine conditions. Immun Inflamm Dis 2017; 5(2):132-140.

40. Schwanke T1, Carragee E, Bremberg M, Reisacher WR. Quality-of-life outcomes in patients who underwent subcutaneous immunotherapy and sublingual immunotherapy in a real-world clinical setting. Am J Rhinol Allergy 2017; 31(5):310-316.

41. Ciprandi G, Cadario G, Valle C, et al. Sublingual Immunotherapy in Polysensitized Patients: Effect on Quality of Life. J Investig Allergol Clin Immunol 2010; 20(4):274-279.
42. Global Initiative for Asthma. Global Strategy for Asthma Management and Prevention, 2018. Available from https:/ginasthma. org 2018.

43. Abramson MJ1, Puy RM, Weiner JM. Injection allergen immunotherapy for asthma. Cochrane Database Syst Rev 2010; 4(8):CD001186.

44. Filanowicz M, Szynkiewicz E, Cegła B, Bartuzi Z. Analysis of the quality of life of patients with asthma and allergic rhinitis after immunotherapy. Postepy Dermatol Alergol 2016; 33(2):134-141. 